\title{
Kernos
}

Revue internationale et pluridisciplinaire de religion grecque antique

$24 \mid 2011$

Varia

\section{Michel CARTRY, Jean-Louis DURAND, Renée KOCH PIETTRE (éds), Architecturer l'invisible. Autels, ligatures, écritures}

\author{
Vinciane Pirenne-Delforge
}

URL : http://journals.openedition.org/kernos/1997

DOI : 10.4000/kernos.1997

ISSN : 2034-7871

\section{Éditeur}

Centre international d'étude de la religion grecque antique

Édition imprimée

Date de publication : 1 janvier 2011

Pagination : 361

ISSN : 0776-3824

\section{Référence électronique}

Vinciane Pirenne-Delforge, « Michel cartry, Jean-Louis duRAnd, Renée кoch PIETTRE (éds), Architecturer l'invisible. Autels, ligatures, écritures », Kernos [En ligne], 24 | 2011, mis en ligne le 18 octobre 2011, consulté le 21 septembre 2020. URL : http://journals.openedition.org/kernos/1997 ; DOI : https:// doi.org/10.4000/kernos. 1997 
Michel CARTRY, Jean-Louis DURAND, Renée KOCH PIETTRE (éds), Architecturer l'invisible. Autels, ligatures, écritures, Turnhout, Brepols, 2009. 1 vol. 15,5 × $24 \mathrm{~cm}$, 448 p. (Bibliothèque de l'École des Hautes Études, Sciences religieuses, 138). ISBN : 9782-503-53172-4.

Ce volume résolument interdisciplinaire offre une réflexion passionnante sur la manière dont des cultures très diverses ont mis en présence des instances considérées comme supérieures, comment elles ont "architecturé l'invisible», pour reprendre le très beau titre du volume. Se revendiquant d'une forme d'empirisme dans le comparatisme, essentiellement fondé sur le dialogue serré entre des spécialistes de domaines différents, les éditeurs offrent une copieuse introduction, qui permet de relier entre elles les interventions qui se répartissent en trois grandes parties : «Ouvrir », « EEuvrer», «Écrire », trois verbes qui sont vus comme les trois modalité de cette « architecture » en train de se faire, quitte à passer par le « défaire ».

De la Mésopotamie à différentes régions de l'Afrique, en s'arrêtant en Grèce et dans le monde chrétien médiéval, c'est à une large interrogation sur les catégories de l'histoire des religions comme «sacrifice » ou «offrande » qu'invitent les réflexions très riches sur des dossiers précis. Celui ou celle qui étudie la religion grecque est évidemment vert(e) de jalousie devant la précision atteinte par la description d'un rituel africian saisi sur le vif par l'anthropologue de terrain. Il ou elle pressent aussi la difficulté, dans son propre domaine, de chercher le sens des manipulations dont une tradition littéraire ou épigraphique fragmentée n'a conservé que des lambeaux. Mais justement, c'est là que le comparatisme pensé dans la lignée des travaux d'équipe pilotés par M. Detienne - un patronage ici assumé - peut aider à poser d'autres questions à un matériel certes très éclaté mais qu'un regard décentré peut apprendre à voir autrement.

Les hellénistes embarqués dans cette aventure comparatiste ont laissé de belles réflexions, qu'un lecteur curieux pourra mettre en résonance avec les études d'autres domaines de recherche: D. Jaillard sur les espaces hermaïques du sacrifice, I. Patera sur les offrandes durables dans l'espace sacrificiel, E. Barra sur les procédures mantiques à Delphes, G. Pironti sur le «miracle » de l'autel d'Aphrodite à Éryx, M. Carastro sur les katadesmoi et les instances de l'enchaînement, R. Koch Piettre sur les manipulations liées aux serments dans la tragédie, et B. Mezzadri sur le sacrifice des rois atlantes conçu par Platon.

Vinciane Pirenne-Delforge

(F.R.S.-FNRS - Université de Liège)

\section{Actes de colloques, ouvrages collectifs et anthologies}

Jan N. Bremmer, Andrew ErSkINe (éds), The Gods of Ancient Greece. Identities and Transformations, Edinburgh, Edinburgh University Press, 2010 (Edinburgh Leventis Studies, 5).

Jan N. Bremmer, Introduction: The Greek Gods in the Twentieth Century, p. 1-18; Albert Henrichs, What is a Greek God?, p. 19-39; Ian Rutherford, Canonizing the Pantheon: the Dodekatheon in Greek Religion and its Origin, p. 43-54; Fritz Graf, Gods in Greek Inscriptions: Some Methodological Questions, p. 55-80; Richard Buxton, Metamorphoses of Gods into Animals and Humans, p. 81-91; Stella Georgoudi, Sacrificing to the Gods: Ancient Evidence and Modern Interpretations, p. 92-105; Anja Klöckner, Getting in Contact: Concepts of Human-Divine Encounter in Classical Greek. Art, p. 106-125; Kenneth Lapatin, New Statues for Old Gods, p. 126-151; Judith M. Barringer, Zeus at Olympia, p. 155-177; Richard Seaford, Zeus in Aeschylus: the Factor of Monetization, p. 178192; Jan N. Bremmer, Hephaistos Sweats or How to Construct an Ambivalent God, p. 193-208; Ivana Petrovic, 